the bacterial and algal slimes and mire of their environment.

G. E. KAISER

R. N. DOETSCH

Department of Microbiology,

University of Maryland,

College Park, Maryland 20742

1 Cox, P. J., and Twigg, G. I., Nature, 250, 260-261 (1974).

2 Schneider, W. R., and Doetsch, R. N., J. Bact., 117, 696-70i (1974).

Nauman, R. K., Holt, S. K., and Cox, C. D., $J$. Bact., 98, 264-279 (i969).

4 Jahn. T. L.. and Landman, M. D., Trans. Am. microsc. Soc., 84. 395-406 (1965).

\section{Function of cochlear hair cells}

RYAN and Dallos' reported the effect of the ototoxic drug kanamycin on behavioural audiograms of chinchillas. In most cases, histological examination with the light microscope showed that $100 \%$ of outer hair cells (OHCs) were missing from much of the basal turn of the cochlea, whereas the row of inner hair cells (IHCs) seemed to be intact. They found quite a good rank order correlation between the extent of $\mathrm{OHC}$ damage and the frequency at which the drug-induced threshold shift begins. In two of the cases they illustrated, however, major discrepancies are obvious. In one case, the threshold shift begins at $3-4 \mathrm{kHz}$ yet $100 \%$ of OHCs are present up to the $7 \mathrm{kHz}$ region. Their whole thesis of facilitation of IHC function by the OHCs depends on the stated assumption that $\mathrm{IHCs}$ remaining in the basal turn, which appear normal under the light microscope, are functionally normal. Yet in this case, there are OHCs scored as intact but in the $4 \mathrm{kHz}$ or $7 \mathrm{kHz}$ region are obviously functionally abnormal. It seems unjustified to assume that IHCs in these regions are similarly not functionally impaired, and to minimise the importance of cytoplasmic changes visible only under the electron microscope. As Wërsall ${ }^{2}$ has noted "... it is remarkable how well preserved the cuticle and the hairs might be in a cell with advanced cellular protoplasmic degeneration."

Furthermore, although I agree with Ryan and Dallos that species differences may be important, it is pertinent to discuss the study of Kiang et al. ${ }^{3}$ in this regard. Using the same drug, they produced almost identical lesions in the cat-massive to complete loss of OHCs and 'intact' IHCs. Yet, in recording VIIIth nerve unit activity, they were unable to detect any units which would respond to frequencies found in these regions of the cochlea. Instead, nonspontaneous, non-responding units were detected (using electrical stimuli) in the high frequency part of the nerve. Here, where there were 'intact' IHCs only, the thresholds were increased beyond the range of their equipment $(80-100 \mathrm{~dB}$ shift). Obviously, these lHCs were functionally abnormal. It is clear that, in contrast to Kiang's cats, Ryan and Dallos' chinchillas could actually hear these frequencies. It seems unreasonable, however, to assume that this detection was mediated by functionally normal IHCs. The hypotheses of IHC and $\mathrm{OHC}$ function based on their arguments, although attractive, are correspondingly weakened in the absence of sufficient proof of the viability of the IHCs in their kanamycin-treated animals.

\section{Geoffrey A. Manley}

Department of Biology,

McGill University.

Montreal, $P Q$.

Canada $\mathrm{H} 3 \mathrm{C} 3 \mathrm{Gl}$

1 Ryan, A., and Dallos, P.. Nature, 253. 44-46
(1975)., J., in Basic Mechanisms in Hearing (edit.
2 Wërsall, Joler, A. R.) 235 (Academic. New York,
by Morler, A.
1973 ). N. Y.-S., Moxon, E. C., and Levine, R. A.,
3 Kiang, N.
in Sensorineural Hearing Loss (edit. by Wolsten-
holme, G. E. W., and Knight, J.), 241 (Churchill,
London, 1970).

Ryan AND Dallos RePly-Manley' presents a criticism of our suggestion ${ }^{2}$ that outer hair cells (OHCs) may facilitate inner hair cell (IHC) function at low sound levels on the basis that our assumption pertaining to the normality of those IHCs remaining in our preparations is incorrect. His arguments are based on two issues one of which, we feel, is inadmissible, the other irrelevant.

First, he cites the less than perfect correlation between extent of OHC lesion and cutoff frequency of hearing loss as a critical weakness. In fact, such correlations are by nature less than exact, because one can only rely on average relationships between cochlear location and corresponding frequency (such frequency maps are not even available for chinchillas). Because of the variability of the cochlear length (about $2 \mathrm{~mm}$ variability in chinchillas) and as hair cell counts progress from apex to base, it is hazardous to assume, as Manley does, that in an individual animal one can uniquely determine the exact locaton of a given, relatively high, frequency. In other words, Manley's objection would have merit only if an accurate frequency map were available for each animal, and if on that basis discrepancies were still found to exist.

The second objection, namely that in the single unit work of Kiang et al. ${ }^{3}$ on the cat, damage patterns 'similar' to ours resulted in greater losses-thus our animals could not have had functioning IHCs-simply lacks a logical basis. Aside from possible species differences that make comparisons tenuous, one must ask the question: What is the best means of establishing that a preparation functions? Clearly, to assess the degree of damage to the hearing organ, one may be on safer grounds by asking what the animal can hear, not by merely studying morphology or the responses of single neurones. Accordingly, in our animals the removal of OHCs resulted in a threshold shift of between 40 and $50 \mathrm{~dB}$. This shift was maintained in magnitude but extended over differing frequency bands which were correlated with the configuration of OHC loss. One would think that variable damage to the IHCs would have resulted in different degrees of loss not merely the same loss covering different extents of the high frequency range.

Finally, Manley's quote from Wersäll's paper is out of context. Wersäll emphasised the poor correlation between signs of damage seen in light and electron microscopy, when such comparisons are made soon after the antibiotic insult to the cochlea. In fact, there is reason to believe that originally damaged cells disappear in time, and that after long survival (as is the case in our study) the surface preparation technique would give a fair indication of the state of the hair cells.

\section{Evanston, Illinois 60201}

1 Manley, G. A., Nature, 255, 657 (1975)

Ryan, A., and Dallos, P., Nature, 253, 44-46 (1975). Kiang, N. Y.-S., Moxon, E. C., and Levine, R. A. in Sensorineural Hearing Loss (edit. by Wolsten in Sensorineural Hearing Loss (edit. 21 (Churchill, London, 1970).

4 Wersäll, J., in Basic Mechanisms in Hearing (edit. by Moller, A. R.), 235 (Academic, New York. 1973).

\section{$\theta$-Positive cells in nude mice born from homozygous $\mathrm{nu} / \mathrm{nu}$ mother}

Two types of $\theta^{+}$lymphocytes have been found in the lymphoid tissues of athymic nude mice. Some have a density of $\theta$ similar to that of peripheral $\mathrm{T}$ lymphocytes of normal mice and make up about $1 \%$ of nude mouse spleen cells ${ }^{1,2}$. Others have a low density of $\theta$ and make up about $20 \%$ of nude mouse spleen cells ${ }^{2,3}$.

Nude mice studied so far were from heterozygous $n u /+$ parents and as has been pointed out ${ }^{3,4}$ there may be an influence of maternal and normal littermate $T$ cells or thymic humoral factors on the cells of the nude littermate.

Litters from homozygous $\mathrm{nu} / \mathrm{nu}$ mothers usually do not survive. We recently had the opportunity, however, to study three nude mice born from a homozygous nu/nu mother and an heterozygous father and nursed by the mother. We found that their content of both strong and weak $\theta$-positive lymphocytes was comparable with that of their mother and $n u / n u$ mice from heterozygous $n u / t$ mothers ${ }^{2,3}$. The results are not completely unequivocal as one 